\title{
Caracteristicas cognitivas y oculares en enfermedad de Alzheimer
}

\author{
Cognitive and ocular characteristics in Alzheimer's disease
}

Garzón P. Sandra Johanna ${ }^{1}$; Camacho M. Marcela²; Tapiero L. Jessica Andrea ${ }^{3}$; Reina Karen Daniela ${ }^{3}$

\section{Resumen}

La enfermedad de Alzheimer/AD es un trastorno neurodegenerativo progresivo que afecta la memoria y todas las funciones cognitivas con una edad de inicio tardío o precoz, y se presenta con una muy baja frecuencia por causa genética por alteración en el gen de la PPA, PS1 O PS2. El elemento etiológico mayor conocido es genético, con múltiples factores de susceptibilidad en interacción con factores medioambientales. Las guías de diagnóstico para la $\mathrm{AD}$ incluyen evaluaciones psicológicas, psiquiátricas y neurológicas con función cerebral, y no incluyen estudios de la función visual como parte del protocolo diagnóstico, siendo fuerte la evidencia de cambios oculares en retina y en algunas funciones visuales que aparecen aun sin el deterioro cognitivo característico de esta enfermedad. Objetivo. Describir las características cognitivas y oculares en la enfermedad de Alzheimer. Metodología. Se realizó una revisión documental de literatura científica en las bases de datos PubMed, Science Direct, Hinari y Ebsco Ebsco, Proquest, entre otras, con un periodo de búsqueda de los últimos 10 años, mediante los términos mesh "Alzheimer Disease and ocular changes", "visual cognitive alteration in Alzheimer" "retina and Alzheimer disease". Resultados: La AD presenta un proceso neurodegenerativo con deterioro cognitivo, que se presenta en todas las regiones de la corteza cerebral, iniciándose en corteza del hipocampo y amígdala cerebral desde donde progresa a la circunvolución para-hipocampal, lóbulos temporales y frontales. Conclusiones. Varios estudios han demostrado que la AD presenta alteraciones en memoria, lenguaje, orientación visoespacial, acompañada por cambios estructurales en cerebro y en la retina al reducir el espesor de las células ganglionares, de las capas de fibras nerviosas y al contener cuerpos de inclusión con proteína beta amiloide $(A \beta)$ y demuestran además que el diagnóstico de alteraciones funcionales por la acumulación de $\mathrm{A} \beta$ es un marcador precoz de la AD.

Palabras claves: Enfermedad de Alzheimer, alteración cognitiva visual en Alzheimer, retina y Alzheimer.

\footnotetext{
1. PhD (c) Universidad de Valencia, España. MSc. Pharm. Universidad Nacional de Colombia. Optómetra, Universidad de la Salle, Colombia. Profesora e investigadora, Facultad de Ciencias de la Salud, Universidad de la Salle.

2. Ms, Universidad de la Salle, Optómetra Universidad de la Salle. Profesora e investigadora, Facultad de Ciencias de la Salud, Universidad de la Salle.

3. Optómetra, Universidad de la Salle. Jóvenes investigadoras asociadas al proyecto de investigación Efectos del entrenamiento visual en la función visual en pacientes con enfermedad de Alzheimer 


\begin{abstract}
Alzheimer's disease / $\mathrm{AD}$ is a progressive neurodegenerative disorder that affects memory and all cognitive functions with a late or early onset age, and occurs with a very low frequency due to genetic causes due to alteration in the PPA gene.,PS1 or PS2. The largest known etiological element is genetic, with multiple susceptibility factors in interaction with environmental factors. Diagnostic guidelines for $\mathrm{AD}$ include psychological, psychiatric and neurological evaluations with brain function, and do not include studies of visual function as part of the diagnostic protocol, with strong evidence of ocular changes in the retina and in some visual functions that appear even without the cognitive deterioration characteristic of this disease. Objective. To describe the cognitive and ocular characteristics in Alzheimer's disease. Methodology. A documentary review of scientific literature was made in the databases PubMed, Science Direct, Hinari and Ebsco Ebsco, Proquest, among others, with a search period of the last 10 years, through the terms mesh "Alzheimer Disease and ocular changes "," visual cognitive alteration in Alzheimer "” retina and alzheimer disease ". Results: AD presents a neurodegenerative process with cognitive deterioration, which occurs in all regions of the cerebral cortex, beginning in the cortex of the hippocampus and cerebral tonsil where it progresses to the para-hippocampal gyrus, temporal and frontal lobes. Conclusions. Several studies have shown that AD presents alterations in memory, language, visuospatial orientation, accompanied by structural changes in brain and retina by reducing the thickness of ganglion cells, the layers of nerve fibers and containing inclusion bodies with protein beta amyloid $(A \beta)$ and further demonstrate that the diagnosis of functional alterations due to the accumulation of $A \beta$ is an early marker of $A D$.
\end{abstract}

Keywords: Alzheimer Disease, visual cognitive alteration in Alzheimer, retina and Alzheimer.

\section{Introducción}

La enfermedad de Alzheimer AD (siglas en inglés) es un complejo proceso neurodegenerativo con múltiples etiologías, se caracteriza por una acumulación progresiva de péptidos $\beta$-amiloide $(A \beta)$, perdida neuronal y deficiencias cognitivas (1). Esta enfermedad afecta aproximadamente al 10\% de los individuos de 65 años o más, afectando la calidad de vida en los adultos mayores y su prevalencia aumenta con el tiempo; clínicamente se caracteriza por una afectación cognitiva y social, y patológicamente se conoce por una acumulación de placas neuronales $\beta$-amiloides (NP) y compromete los ovillos neurofibrilares (NTF) en el cerebro.(1-3) La deposición de la placa $A \beta$ se asocia con una disfunción de la red sináptica transversal, una atrofia cerebral progresiva y un deterioro cognitivo longitudinal.(4)
La patología de $\mathrm{AD}$ incluye depósitos cerebrales de agregados anormales en forma de placas seniles (SP), anormalmente fosforilados en forma de enredos neurofibrilares (NFT), que se observan en estados avanzados de la enfermedad(5). Estudios recientes de Calebresi y colaboradores, 2015 han evidenciado la presencia de marcadores neurodegenerativos tempranos a nivel cerebral y retinal, cambios en la vasculatura y presencia de fibras peptídicas en las capas retinales con una reducción de agudeza visual, sensibilidad al contraste, percepción de color, percepción de movimiento construcción espacial y memoria visual $(4,6,7)$.

Como se mencionó anteriormente la $\mathrm{AD}$ es un trastorno neurodegenerativo progresivo que afecta la memoria y todas las funciones cognitivas, estos se dividen generalmente en memoria, lengua, atención y funcionamiento visoespacial, que a su vez 
presentan síntomas visuales que son prominentes debido a que la patología se localiza en la región parieto-occipital, donde se refiere como la variante visual de la enfermedad de $\operatorname{Alzheimer}(4,7)$. Es importante destacar que la progresión neuropatológica ocurre tanto en el ojo como en el cerebro, y se han observado múltiples cambios visuales; mostrando una prevalencia en el mundo en un 5\%, con una edad de inicio tardío o precoz, y se presenta con una muy baja frecuencia por causa genética por alteración en el gen de la PPA, PS1 O PS2. La mayoría de casos están influenciados por una genética compleja con múltiples factores de susceptibilidad en interacción con factores medioambientales, pero en aproximadamente un $10 \%$ de los pacientes se manifiesta en forma familiar (8).

\section{Características genéticas}

La AD se relaciona frecuentemente con susceptibilidad genética que proporciona información probabilística sobre el riesgo de padecer la enfermedad (9); las pruebas genéticas han identificado tres loci genéticos: proteína precursora amiloide (APP), presenilina-1 (PSEN1) y presenilina-2 (PSEN2) como genes de susceptibilidad para la AD temprana y SORL1 y APOE para la AD de inicio tardío; aunque estos genes son útiles para predecir el riesgo de desarrollar la enfermedad, su falta de especificidad, sensibilidad diagnóstica y la influencia de factores ambientales externos los hacen inadecuados como biomarcadores (4).

La progresión de pérdida neuronal y la posterior atrofia cerebral progresiva, se asocian con menores concentraciones de oxiesteroles aumentando la concentración de 24S-Hidroxicolesterol, que es un producto del metabolismo del colesterol cerebral, el cual representa un marcador sustituto de atrofia cerebral en esta enfermedad neurodegenerati$\mathrm{va}(10)$. Este hallazgo se encuentra en aproximadamente $50 \%$ de los pacientes con $\mathrm{AD}$, causando interrupción de la circulación y generando como consecuencia atrofia cerebral (11).
Además, como característica principal en la $\mathrm{AD}$ se destaca la acumulación de placas neuronales $\beta$-amiloides; este es un producto fisiológico del metabolismo celular que se genera a través de sucesivas etapas de ruptura de la proteina precursora amiloide (APP) por $\beta$ - y $\gamma$-secretasa. Es probable que el evento de inicio en la patogénesis en la $\mathrm{AD}$ sea el desequilibrio entre la producción y el aclaramiento de $A \beta$ (12). De esta manera las cantidades excesivas de $A \beta$ solubles e hidrófobos se unen directamente a diferentes componentes de membranas plasmáticas neuronales y no neuronales dentro del SNC, desencadenando múltiples mediadores moleculares que afectan diversas vías intracelulares que comprometen en última instancia la disfunción sináptica y la degeneración neuronal (12-15).

De forma similar al cerebro, las inclusiones de la proteína Tau y el depósito de $\mathrm{A} \beta$ se han descrito en la retina de pacientes con $\mathrm{AD}$ y en modelos animales de la enfermedad. Los ratones transgénicos portadores de la mutación tau P301S humana contienen agregados de tau en la retina, y presentan déficits funcionales de las células ganglionares de la retina, mayor susceptibilidad al daño excitotóxico y alteración de la señalización del factor neurotrófico. La disfunción Tau ha sido bien descrita en la $\mathrm{AD}$ por algunos autores. En la actualidad, se sabe poco sobre la secuencia temprana de eventos que conducen a la patología tau en la $\mathrm{AD}$, destacando la necesidad de dilucidar la interacción de los cambios moleculares y celulares durante las etapas pre-sintomáticas de la enfermedad (16).

De acuerdo con lo anterior, para el diagnóstico actual de $\mathrm{AD}$ con propósitos de investigación se requiere evidencia de 1) depósito de amiloide, bajas concentraciones de $\beta$-amiloide en líquido cefalo raquídeo (LCR), o depósito de $\beta$-amiloide en tomografía por emisión de positrones (TEP) 2) neuro-degeneración por autopsia, altas concentraciones de Tau fosforilado (taup) en LCR, y patrón de atrofia tipo EA en las imágenes estructurales. 
Sin embargo, en la práctica clínica habitual los bio-marcadores amiloide y tau no son requeridos para el diagnóstico (17).

\section{Características epidemiológicas}

En Colombia, se tiene la más alta prevalencia de AD del mundo, en Yarumal, municipio de Antioquia, donde se han detectado casos por desórdenes de genética familiar. Aun existiendo en Colombia la política nacional de envejecimiento y vejez que plantea el diseño, difusión y aplicación de metodologías de planificación y gestión pública que promuevan una efectiva realización de los derechos a las personas mayores como sujetos de especial protección (18), es limitado el estudio para diagnóstico de los desórdenes cognitivos - comportamentales que causan perdida en la calidad de vida del paciente con $\mathrm{AD}(19)$.

Para los casos de Alzheimer a nivel nacional se estima que la cifra de demencia en adultos mayores en Colombia es de 1.8 y $3.4 \%$ entre 65 y 75 años respectivamente, se calcula que el tiempo de sobrevida de un paciente con enfermedad de Alzheimer es entre 5 y 10 ańos en promedio, el cual varía de acuerdo a la edad de aparición (20). En Colombia según el "Estudio Nacional de Salud Mental Colombia 2003" se estimó que el Alzheimer se incluye dentro de las enfermedades neuropsiquiátricas que generan discapacidad, con mayor incidencia en Bogotá y Medellín (21). En Colombia, al igual que en otros países latinoamericanos como Ecuador, Cuba, Perú, Uruguay, Venezuela, Argentina y México, se ha presentado un crecimiento importante de estas enfermedades en los últimos 20 años que impactan un deterioro cognitivo y calidad de vida de la población (22).

Según datos de la Organización panamericana de salud del ańo 2012, el incremento de la población adulta mayor en América latina y el Caribe es inminente y se estimó en el año 2010, alrededor de 35.6 millones de personas en todo el mundo con demencia, cifra que se duplicará cada 20 ańos, es decir, cerca de 65.7 millones para el 2030 (23). De esta manera, a medida que envejece la población aumentan las enfermedades crónicas no transmisibles y neurodegenerativas(20) (3). En los Estados Unidos se estima que afecta a más de 5 millones de personas, con un aumento esperado a 13 millones para el año 2050. La presentación clínica es la pérdida progresiva de memoria y la función cognitiva, que en última instancia conduce a una pérdida de independencia y causa un gran costo personal en el paciente y la familia. Los costos de cuidado de pacientes con enfermedad de Alzheimer en 2010 se estimaron en más de \$ 172 mil millones en EEUU, un costo anual que se predice para aumentar a un billón de dólares en 2050 a menos que se desarrollen tratamientos modificadores de la enfermedad (24).

A nivel mundial, existen cerca de 46 millones de personas que viven con demencia, principalmente demencia senil que es muy difícil diferenciarla de $\mathrm{AD}$ (4). Las alteraciones cognitivas y alienación social en este tipo de enfermedades, además de los daños funcionales como el visual, la convierten en una enfermedad de interés en salud pública, en la cual la intervención en el diagnóstico oportuno y el conocimiento de las características de la enfermedad, pueden contribuir en el adecuado direccionamiento en las entidades de salud como en el mejoramiento de la calidad de vida de los pacientes. Los estudios epidemiológicos han demostrado que la actividad física tiene un efecto beneficioso sobre la salud del cerebro, a través de la activación de mecanismos que incrementan la plasticidad cerebral, normalizan la vascularización cerebral, estimulan la neurogénesis, reducen los niveles de inflamación o incluso logran reducir la placa amiloide (25).

\section{Deterioros cognitivos}

Los desórdenes cognitivos, comportamentales y pérdida en la calidad de vida del paciente con enfermedad de Alzheimer se producen por las altera- 
ciones neurodegenerativas causadas la acumulación de péptidos $\beta$-amiloide. La agrupación de placas extracelulares de amiloide se producen por la insolubilidad de más de 42 aminoácidos que se agrupan formando estas placas. El proceso patológico al interior de las neuronas, continúa con incremento de la proteína Tau, encargada de estabilizar los neurotúbulos, la cual es fosforilada de un modo excesivo, con lo que se altera la integridad de los neurotúbulos neuronales y se producen unos agregados intraneuronales denominados ovillos neurofibrilares (1). Los desórdenes cognitivos y la demencia en esta enfermedad, se relacionan con cambios microvasculares en el tejido cerebral, principalmente en la materia blanca, que produce disfunción neurológica $(26,27)$. Es por este motivo que los pacientes con $\mathrm{AD}$, son más propensos a presentar cambios estructurales en la microvasculatura retinal, que reflejan cambios similares dentro de la microcirculación cerebral (26).

Recientes estudios del año 2017 de Croteau y colaboradores, justifican el deterioro cognitivo en la $\mathrm{AD}$ con alteraciones en el metabolismo de la glucosa cerebral impidiendo el metabolismo de la cetona encefálica y causando atrofia cerebral (27). Por otro lado, Epperly y otros investigadores, en el ańo 2015 asocian el deterioro cognitivo con incrementos en la colinesterasa y la memantina, la cual es susceptible de modificar a través de medicamentos que reducen la colinesterasa, aumentando así acetilcolina y mejorando el deterioro cognitivo y funcional en pacientes con enfermedad de Alzheimer (28); Estudios también relacionan la demencia con enfermedades cerebrales como la disfunción capilar debido a la disponibilidad de oxígeno que aborda gradualmente las necesidades metabólicas del cerebro causando deterioro neurológico (29).

Se cree que la pérdida normal de la memoria relacionada con la edad comienza en la circunvolución dentada del hipocampo. Esta observación está respaldada por exploraciones cerebrales con resonancia magnética funcional (fMRI) de alta resolución y estudios cognitivos. En la $\mathrm{AD}$, la pérdida neuronal en la corteza entorrinal (que detalla la codificación cerebral de la memoria a largo plazo) preexiste por un largo período de déficits en la conectividad de la formación del hipocampo, donde hay una disminución dependiente de la edad en el número de nuevas neuronas y del mismo modo, hay una disminución de la neurogénesis. Los procesos anteriores se incrementan con la edad, por cambios en la estructura sináptica, con reducción de la densidad de los contactos sinápticos formados en las células granulares lo que produce déficit en la neurogénesis que contribuye a las deficiencias cognitivas (30).

\section{Clasificación Alzheimer}

Al ser una enfermedad heterogénea la $\mathrm{AD}$ tiene múltiples subtipos cognitivos ya que en estos pacientes se ha reducido la función colinérgica(28). Es importante tener en cuenta que el deterioro cognitivo leve en ocasiones es una condición previa a la demencia, es decir que el porcentaje de deterioro cognitivo leve a desarrollar Alzheimer es de 12\% (22). En la tabla 1 se muestran las diferentes clasificaciones para la $\mathrm{AD}$, siendo la más utilizada la clasificación propuesta por Mormino E., Papp K. en el año 2016.

El diagnóstico de la $\mathrm{AD}$ en su fase pre-demencia es de vital importancia, no sólo para la provisión de mejor cuidado clínico, sino también a la luz de la llegada anticipada de tratamientos modificadores de la enfermedad para la $\mathrm{AD}$, dado que este tipo de tratamientos es probable que sean más eficaces si se aplican en etapas anteriores de la enfermedad (31). 
Tabla 1. Clasificación de AD.

\begin{tabular}{|c|c|c|c|}
\hline Autores & Ańo & Clasificación & Descripción \\
\hline $\begin{array}{l}\text { Sid E., Stephen C. Munro } \\
\text { Cullum, Staging Dementia } \\
\text { Using Clinical Dementia } \\
\text { Rating Scale Sum of Boxes } \\
\text { Scores A Texas Alzheimer's } \\
\text { Research Consortium Study } \\
\text { (32): }\end{array}$ & 2008 & $\begin{array}{l}\text { - Deterioro leve } \\
\text { - Deterioro moderado } \\
\text { - Deterioro severo }\end{array}$ & $\begin{array}{l}\text { Se evalúa en } 6 \text { dominios de funcionamiento: } \\
\text { memoria, orientación, juicio y solución de } \\
\text { problemas, asuntos comunitarios, hogar y } \\
\text { pasatiempos, y cuidado personal. Cada dominio } \\
\text { se califica en una escala de funcionamiento de } 5 \\
\text { puntos } \\
\text { 0-Sin degradación } \\
\text { 0.5-Deterioro cuestionable } \\
\text { 1-Deterioro leve: Memoria } \\
2 \text {-Deterioro moderado: Orientación, juicio y } \\
\text { solución de problemas } \\
\text { 3- Deterioro severo: Asuntos comunitarios, hogar y } \\
\text { pasatiempos, y cuidado personal. }\end{array}$ \\
\hline $\begin{array}{l}\text { Lopera Restrepo F. La } \\
\text { enfermedad de Alzheimer } \\
\text { Familiar (8) }\end{array}$ & 2012 & $\begin{array}{l}\text { - AD Temprana } \\
\text { - AD Tardía }\end{array}$ & $\begin{array}{l}\text {-AD Temprana: Pérdida progresiva de la memoria } \\
\text { es el primer hallazgo y el más frecuente, también } \\
\text { se presentan trastornos del lenguaje, lentitud } \\
\text { en el procesamiento cognitivo, problemas de } \\
\text { atención, temblor, parkinsonismo, características } \\
\text { extrapiramidales, mioclonías y convulsiones. } \\
\text {-AD tardía: Presenta gen de la proteína precursora } \\
\text { de amiloide (PPA), el gen de la presenilina } 1 \text { y el } \\
\text { gen de la presenilina 2. se presentan trastornos del } \\
\text { lenguaje, lentitud en el procesamiento cognitivo, } \\
\text { problemas de atención, temblor, parkinsonismo, } \\
\text { características extrapiramidales, mioclonías y } \\
\text { convulsiones (8). }\end{array}$ \\
\hline $\begin{array}{l}\text { Soto et al. Mar J, Soto-Gordoa } \\
\text { M, Arrospide A, Moreno- } \\
\text { Izco F, Martínez-Lage P. } \\
\text { "Fitting the epidemiology and } \\
\text { neuropathology of the early } \\
\text { stages of Alzheimer's disease to } \\
\text { prevent dementia"(33) }\end{array}$ & 2015 & $\begin{array}{l}\text { - AD preclínica } \\
\text { - } \mathrm{AD} \text { moderada } \\
\text { - AD con demencia } \\
\text { (tardío) }\end{array}$ & $\begin{array}{l}\text {-AD Preclínica: Síntomas clínicos } \\
\text {-AD moderada: Cambios patológicos previos, en } \\
\text { relación con nuestro uso de la deposición de Aß. } \\
\text { En su caso la etapa moderada muestra los mismos } \\
\text { síntomas cognitivos y psicológicos severos al igual } \\
\text { que su fase tardía } \\
\text {-AD Demencia: Signos cognitivos y psicológicos } \\
\text { severos. }\end{array}$ \\
\hline $\begin{array}{l}\text { Chan et al., Gallaher, L. M., } \\
\text { Moodley, K., Minati, L., } \\
\text { Burgess, N., Hartley, T. The } 4 \\
\text { Mountains Test: A Short Test } \\
\text { of Spatial Memory with High } \\
\text { Sensitivity for the Diagnosis } \\
\text { of Pre-dementia Alzheimer's } \\
\text { Disease. (31) }\end{array}$ & 2016 & $\begin{array}{l}\text {-Predemencia (etapa } \\
\text { clínicamente silenciosa } \\
\text { presintomático). } \\
\text { •Etapa sintomática } \\
\text { / "prodrómica", se } \\
\text { manifiesta como } \\
\text { deterioro cognitivo leve } \\
(\mathrm{MCI}) \text {. }\end{array}$ & $\begin{array}{l}\text {-En la etapa de predemencia: Los síntomas son poco } \\
\text { notorios. } \\
\text {-En la etapa sintomática: los individuos presentan } \\
\text { deterioro cognitivo (típicamente deterioro de la } \\
\text { memoria) pero retienen la independencia funcional } \\
\text { y las actividades de la vida diaria conservados. }\end{array}$ \\
\hline $\begin{array}{l}\text { Mormino E., Papp K. } \\
\text { Cognitive Decline in } \\
\text { Preclinical Stage } 2 \text { Alzheimer } \\
\text { Disease and Implications for } \\
\text { Prevention Trials (34) }\end{array}$ & 2016 & $\begin{array}{l}\text { - Etapa } 1 \\
\text { - Etapa } 2 \\
\text { - Etapa } 3\end{array}$ & $\begin{array}{l}\text { Todas las etapas comienzan con una aberrante } \\
\text { acumulación de } A \beta \text {, seguida por una } \\
\text { neurodegeneración (ND) y un posterior deterioro } \\
\text { cognitivo. } \\
\text {-La etapa } 1 \text { con acumulación de } A \beta+\text { y ND-, } \\
\text {-La etapa } 2 \text { con acumulación de } A \beta+\text { y } N D \text {, } \\
\text {-Etapa } 3 \text { son similarmente } A \beta+/ N D+\text { pero } \\
\text { también muestran un deterioro cognitivo sutil. }\end{array}$ \\
\hline
\end{tabular}




\begin{tabular}{|c|c|c|c|}
\hline $\begin{array}{l}\text { Alvarez J. Biomarcadores en la } \\
\text { enfermedad de Alzheimer. }\end{array}$ & 2016 & $\begin{array}{l}\text { - Tipo familiar: que } \\
\text { se transmite de forma } \\
\text { dominante, Autosomal- } \\
\text { onset Alzheimer's } \\
\text { Diisease (ADAD/ } \\
\text { EOAD). } \\
\text { - Forma tardía de la } \\
\text { patología, Late-onset } \\
\text { Alzheimer's Diasease } \\
(\text { LOAD). }\end{array}$ & $\begin{array}{l}\text { EOAD: Mutaciones en los genes APP, PSEN1 y } \\
\text { PSEN2. } \\
\text { LOAD: Altos niveles de Aß42 relacionados con } \\
\text { marcadores sinápticos, deterioros cognitivos (35). }\end{array}$ \\
\hline $\begin{array}{l}\text { Alonso M., Sosa A., Trujillo Z. } \\
\text { Visión actual de las demencias }\end{array}$ & 2016 & $\begin{array}{l}\text { - Curso atípico. } \\
\text { - Presentación } \\
\text { etiológicamente mixta. }\end{array}$ & $\begin{array}{l}\text {-Curso atípico: criterios clínicos básicos en } \\
\text { cuanto a la naturaleza de los déficits cognitivos } \\
\text { para demencia de Alzheimer, pero presenta un } \\
\text { inicio repentino del deterioro cognitivo o existen } \\
\text { insuficientes detalles en la historia clínica o } \\
\text { documentación cognitiva objetiva de deterioro } \\
\text { progresivo. } \\
\text {-Presentación etiológicamente mixta: Cumple con } \\
\text { todos los criterios para demencia de Alzheimer pero } \\
\text { existe evidencia de: } \\
\text { a. Enfermedad cerebrovascular concomitante, } \\
\text { definida por una historia de accidente } \\
\text { cerebrovascular relacionado temporalmente a la } \\
\text { aparición o empeoramiento del deterioro cognitivo, } \\
\text { presencia de múltiples o extensos infartos o severas } \\
\text { hiperintensidades en la materia blanca. } \\
\text { b. Características de la demencia por cuerpos de } \\
\text { Lewy u otras de la demencia. } \\
\text { c. Evidencia de otra enfermedad neurológica o } \\
\text { comorbilidad médica no neurológica; uso de } \\
\text { medicamentos que pudieran tener un efecto } \\
\text { sustancial sobre la cognición (36). }\end{array}$ \\
\hline
\end{tabular}

Fuente. Elaboración propia.

\section{Hallazgos retinales}

Varios estudios han demostrado que la $\mathrm{AD}$ presenta cambios estructurales en la retina al reducir el espesor de las células ganglionares, de las capas de fibras nerviosas y al contener cuerpos de inclusión con proteína beta amiloide $A \beta$ (37) y demuestran además que el diagnóstico de alteraciones funcionales por la acumulación de $A \beta$ es un marcador precoz de la $\mathrm{AD}(33)$.

En la AD se encuentran hallazgos retinales de depósitos de amiloide- $\beta$ en drusas oculares y placas seniles, frecuentemente encontrados también en degeneración macular relacionada con la edad (DMAE) (38). sin embargo en esta última enfermedad no se encuentran asociaciones en cerebro, mientras que en $\mathrm{AD}$ existen cambios análogos cerebrales y retinales (4). La vasculatura cerebral y retiniana comparten orígenes embriológicos similares, características anatómicas, propiedades fisiológicas y mecanismos de regulación, es por esto que los cambios de la retina también se han observado en la $\mathrm{AD}$, donde la $\beta$-amiloide ha sido identificada en la microvasculatura de la retina y la coroides a partir de modelos animales con experimentos preclínicos (39). La tortuosidad es una característica común en arterias y venas frecuentemente asociadas con enfermedad vascular y envejecimiento, pero los mecanismos subyacentes para su iniciación y desarrollo aún no están claros. Se encuentran implicados múltiples factores en el proceso de tortuosidad vascular, incluidos los factores genéticos, la enfermedad vascular degenerativa y la alteración en el flujo sanguíneo y presión, alterando el endotelio vascular, causando microhe- 
morragias y alteraciones la distribución sanguínea a través de la red retiniana (39).

Es importante destacar que la retina está organizada en capas de neuronas especializadas interconectadas por sinapsis; las principales células involucradas en el procesamiento de la retina son los fotorreceptores (conos y bastones), y las células bipolares y ganglionares(4). La retina también comprende las células de la interneurona (células amacrinas y células horizontales) y las células Müller gliales que mantienen las propiedades homeostáticas y metabólicas $(4,40)$. Las células ganglionares, son las encargadas de expulsar los axones que forman el nervio óptico, luego transfieren la señal a la corteza visual mediante la emisión de potenciales de acción(4), entre todas las estructuras de la retina, estas células ofrecen una etapa particularmente interesante para estudiar el funcionamiento cerebral indirectamente, debido a sus propiedades anatómicas y funcionales, así como las características de sus mediciones electrofisiológicas. Además, las células ganglionares de la retina constituyen la última y más integrada capa retiniana y, en las vías visuales, ofrecen un acceso indirecto y fácilmente medible de la función cerebral entre el procesamiento visual de fototransducción en fotorreceptores y el procesamiento visual talámico y cortical (40) (4).Estas células se pueden visualizar a través de tecnología de imágenes de alta resolución como la tomografía de coherencia óptica (OCT), este examen evidencia indirectamente la pérdida de células ganglionares y el espesor de las capas de la retina esto se relaciona con la disfunción visual en los pacientes con AD; La evidencia actual también sugiere que el grosor de la capa de células nerviosas de la retina disminuye a medida que la enfermedad progresa y que existe una correlación significativa entre el volumen total de la mácula y el nivel de deterioro cognitivo medido por el estado mini mental (MMSE) $(4,41)$. Los estudios histopatológicos han sugerido la posible pérdida del grosor de la capa de fibras nerviosas retinianas (CFNR) en la $\mathrm{AD}$ por el agotamiento de las células ganglionares de la retina y los axones del nervio óptico (42). En las investigaciones que utilizaron el OCT, una mayor proporción de pacientes con AD que los sujetos sanos de la misma edad mostraron signos de neuropatía óptica y esto se manifestó como atrofia del disco óptico, ahuecamiento del disco óptico patológico y adelgazamiento del borde neurorretiniano y de la CFNR; Los estudios de datos indican una disminución significativa en la CFNR peripapilar y cambios en el grosor y el volumen macular que es progresivo desde el deterioro cognitivo leve (DCL) en los ojos con $\mathrm{AD}(6)$. Parisi y sus colegas usaron por primera vez el OCT para estudiar un grupo de pacientes con AD y los compararon con un grupo de controles de la misma edad. En pacientes con $\mathrm{AD}$, los resultados del OCT mostraron un grosor reducido de la retina en general y en cada cuadrante. El grosor medio de la CFNR se confirmó que se redujo en pacientes con $\mathrm{AD}$ por varios grupos independientes. $\mathrm{La}$ mayoría de los estudios observaron una reducción significativa del grosor de la CFNR en todos los cuadrantes, pero predominantemente en los cuadrantes superior e inferior(43), Haan J., Verbraak F., Visser P., Bouwman F., encuentran que el grosor de la capa de células ganglionares es menor en estos cuadrantes cuadrantes superior e inferior en particular ya que contienen más neuronas y por lo tanto la neurodegeneración es más marcada. De manera similar se espera un cambio prominente de las células ganglionares más grueso en el anillo interno de la mácula, en contraste de esto, el grosor de la mácula muestra una disminución en el anillo externo, que puede reflejar la pérdida de células de la retina en la periferia (41).

Ong Y'T y colaboradores en el año 2015, descubrieron que las disminuciones de grosor de las capas de la retina se correlacionaban con la disminución del tamańo de las regiones occipital y temporal del cerebro basados en OCT y Resonancia magnética (RM) en una población mayor de 60 años, donde estas correlaciones demuestran que la degeneración de la retina es paralela en regiones específicas del cerebro implicadas en la $\mathrm{AD}(44)$. Entre los cambios retinianos están los vasculares los cuales identifi- 
cados en asociación con AD pueden ofrecer valor tanto para comprender la etiología de la enfermedad como para ayudar al diagnóstico temprano y no invasivo de esta enfermedad, esto teniendo en cuenta que los cambios en el flujo sanguíneo pueden llegar a la muerte celular de la retina. Por otro lado con la ayuda de la oximetría retiniana la cual se usa para detectar cambios en el metabolismo del ojo, se han observado anomalías de la $\mathrm{AD}$, donde la saturación del oxígeno retiniano en arteriolas y vénulas es elevada(4); Otro cambio es el que se presenta en el nervio óptico, que a diferencia de otros nervios craneales, está rodeado de mielina producida por oligodendrocitos (en lugar de células de Schwann que se encuentran en los nervios periféricos), está recubierto por las meninges y tiene los mismos orígenes embrionarios que el sistema nervioso central (SNC). Por lo tanto, el nervio óptico se considera parte del SNC, y como tal, es la única parte directamente visible de él; en esta estructura se encontró disminución de los axones, afectando preferentemente a los axones de gran diámetro. Las mediciones precisas de la cabeza del nervio óptico y la capa de fibras nerviosas retinianas (CFNR) son ahora posibles con los dispositivos modernos como OCT por las ventajas antes mencionadas(45). La medición del grosor de la retina mediante OCT ha generado gran interés, y muchos estudios han informado una disminución significativa del grosor en estos pacientes y parece tener una utilidad potencial en el diagnóstico(42). Sabiendo aún que el grosor de CFNR disminuyen con la edad; incluso se ha descrito la hipótesis de que la $\mathrm{AD}$ y el glaucoma se asocian, ya que comparten la misma fisiopatología como: neuroinflamación, $A \beta$ y muerte de las células ganglionares de la retina, además de esto la prevalencia de glaucoma aumenta en pacientes con $\mathrm{AD}$, con un porcentaje de $25.9 \%$ comparado $1 \%-5.2 \%$ en la población normal. La correlación inversa no es tan clara, ya que algunos estudios de pacientes con glaucoma muestran un mayor riesgo con $\mathrm{AD}$, mientras que otros estudios informan que no hubo asociación, probablemente citan que $\mathrm{AD}$ es un factor de riesgo para glaucoma o quizás solo comparten el mismo proceso fisiopatológico con neurodegeneración retiniana, pero, se destaca la necesidad de tener en cuenta el glaucoma como factor contribuyente al grosor de la retina $(4,41)$. Siendo una hipótesis la correlación de estas mediciones con la estructura y función del SNC, las imágenes oculares pueden proporcionar buena información para el diagnóstico de la enfermedad (45). Algunos estudios han notado anormalidades en la electrofisiología de la capa de la retina, adelgazamiento de la capa de fibras nerviosas retinianas (CFNR) y la cabeza del nervio óptico (42).

Es importante abrir la puerta a la detección de este tipo de demencia mediante el uso de imágenes oculares no invasivas puesto que se informó en algunos estudios que la acumulación de la proteína beta-amiloide $(A \beta)$ es un sello distintivo en la retina de pacientes con Alzheimer. El estudio original de Koronyo-Hamaoui y colaboradores, identificaron la patología del $A \beta$ en las retinas de pacientes con AD definitiva y en estadio temprano; demostrando varios tipos de depósitos, incluyendo placas difusas, inmaduras, maduras y las asociadas con depósitos de lípidos(46). Por otro lado, las células ganglionares y las interneuronas potencialmente retinianas (células horizontales, bipolares y amacrinas) se ven afectadas por las placas de $A \beta$; sin embargo, todas las células retinianas pueden verse comprometidas en etapas posteriores del trastorno. El amiloide $\beta$ ha sido identificado en drusas retinianas, un ejemplo de esto distintivo es la degeneración macular relacionada con la edad (DMRE), una de las principales causas de ceguera en todo el mundo, donde uno de los principales signos son las drusas. Las drusas son depósitos extracelulares anormales a lo largo de la superficie basal del epitelio pigmentado de la retina (EPR), las drusas que contienen $A \beta$ se asocian con la atrofia del EPR y la muerte de los fotorreceptores y curiosamente se ha encontrado que las drusas periféricas están significativamente asociadas con $\mathrm{AD}(4)$.

De acuerdo con la relación de las estructuras de la retina, las celulas ganglionares permiten estudiar el funcionamiento cerebral indirectamente, es por 
ello que los hallazgos patológicos más importantes se encuentran en esta región, pero tambien se han identificado otras alteraciones en estructuras oculares; en la tabla No 2 se observan estos cambios patológicos.

Tabla 2. Cambios patológicos ocualres de AD.

\begin{tabular}{|l|l|}
\hline Estructura ocular & \multicolumn{1}{|c|}{ Cambios patológicos en AD } \\
\hline Cristalino & $\begin{array}{l}\text { Depósitos de } \beta \text {-amiloide forman } \\
\text { opacidades }\end{array}$ \\
\hline Retina & $\begin{array}{l}\text { Reducción de las células ganglionares de } \\
\text { la retina, adelgazamiento de la capa de } \\
\text { células nerviosas, principalmente celulas } \\
\text { ganglionares (4,40) }\end{array}$ \\
\hline Disco Óptico & $\begin{array}{l}\text { Palidez de disco, atrofia óptica, ventosas } \\
\text { (muesca del disco óptico); adelgazamiento } \\
\text { del borde neurorretiniano y de la CFNR } \\
\text { (42); En algunos pacientes se han } \\
\text { identificado glaucoma de ángulo abierto } \\
\text { sin previos antecedentes (57) }\end{array}$ \\
\hline Nervio Óptico & $\begin{array}{l}\text { Disminución de los axones de las celulas } \\
\text { ganglionares (42) atrofia del disco ópti }\end{array}$ \\
\hline $\begin{array}{l}\text { Cuerpo geniculado } \\
\text { lateral }\end{array}$ & $\begin{array}{l}\text { Acumulación de lipofuscina asociada } \\
\text { a estress oxidativo y disfunción } \\
\text { mitocondrial (58) }\end{array}$ \\
\hline
\end{tabular}

Fuente. Elaboración autores, basado en Armstrong (57).

\section{Alteración en la función visual}

La Agudeza Visual y la Sensibilidad al contraste consisten en la habilidad de reconocer objetos, con una diferencia de que la sensibilidad al contraste maneja rango de frecuencias espaciales y generalmente se prueba usando gráficos. En los pacientes con AD la Agudeza visual y sensibilidad al contraste son bajas ya que en una mayor prevalencia presentan catarata, por consiguiente, los niveles de luminancia afectan y no reconocen las imágenes, presentan un mayor riesgo de caídas y fracturas. También se ha observado una reducción en la velocidad de lectura, particularmente a menores sensibilidades de contraste y es más evidente con palabras irregulares en el texto. En algunos estudios no se concreta ciertamente el estado de la visión de color en estos pacientes, pero Zara Javaid F., Brenton J., Guo L., Cordeiro M., 2016 descri- ben la afectación depende del ojo afectado, correlacionándolo con cambios visuales de los colores azul-amarillo (retina) y rojo- verde (nervio óptico), también menciona que en estudios anteriores se ha indicado deficiencias sobre el eje Tritan (deficiencia color azul) que se asocia al realizar el examen de estado mini mental (MMSE) (4).

Javaid y colaboradores en el año 2016, encontraron alteraciones en agudeza visual, sensibilidad al contraste, visión cromática, campo visual, percepción de movimiento y estereopsis. También se han descrito déficits relacionados con las vías de procesamiento ventral y dorsal que producen deficiencias en el reconocimiento facial y de objetos, así como en el procesamiento del color y de patrones de formas $(47,48)$.

Kirby E. y colaboradores en el año 2010, en su artículo "Visual impairment in Alzheimer's disease: a critical review.2010", los resultados de su estudio donde arroja que las disfunciones visuales comunes fueron visuoconstructivas (87.3\%), visuoperceptuales (63.6\%), agnosia de objetos (47.3\%), prosopagnosia (45.5\%), alucinación visual (27.3\%) y simultanagnosia (12.7\%). Los síntomas de la disfunción ventral de la vía visual fueron más frecuentes que los de la vía dorsal $(48,49)$.

Los cambios neurodegenerativos y la disfunción sináptica, particularmente debido a la acumulación de $A \beta$ ha provocado la pérdida de campo visual en estos pacientes; Humphrey se ha utilizado en pruebas de campo visual entre pacientes con $\mathrm{AD}$ y controles de la misma edad donde se ha demostrado las pérdidas de sensibilidad en pacientes con AD (4). Por otro lado, Pavisic, et al., 2017 mencionan la atrofia cortical posterior siendo un síndrome neurodegenerativo progresivo causado principalmente por patología AD y caracterizado por una disfunción visuoespacial y visuoperceptual progresiva con memoria, percepción y juicio relativamente conservados. Las personas con atrofia cortical posterior a menudo manifiestan algunas o todas las características del síndrome de Balint, 
como la simultanagnosia, la apraxia oculomotora, la ataxia óptica y la agnosia ambiental (47). Durante las últimas décadas, algunos autores se dieron cuenta de que las disfunciones corticales por sí solas no pueden explicar por completo el patrón de defectos observados. Específicamente, múltiples formas de evidencia apuntan hacia la participación de las células ganglionares de la retina y sus axones en el nervio óptico como base de la disfunción visual en la $\mathrm{AD}$. De hecho, las lesiones histopatológicas asociadas con la pérdida neuronal AD, las placas beta amiloideas, los ovillos neurofibrilares y la degeneración granulovacuolar se han visto no solo en las estructuras cerebrales que históricamente se pensaba que estaban involucradas en la $\mathrm{AD}$, sino también dentro de la neurorretina(43).

El ERG es un potencial en masa (mERG), resultante de la suma de la actividad eléctrica de todas las células de la retina, el cual constituye una técnica clínica bien establecida para evaluar la función retiniana global(50-55). La mayoría de estos estudios han encontrado que las respuestas anomarles de mfERG central y periférica disminuyen con aumento de la edad, pero la disminución es más prominente en la retina central y hallazgos como disminución de la variación naso-temporal $(29,50)$

En la tabla No. 3 se observan los cambios en función visual en los pacientes con $\mathrm{AD}$, que se presentan en diferentes etapas de la enfermedad. Los hallazgos se presentan según la clasificación de Mormino (34).

Tabla 3. Cambios visuales de la AD.

\begin{tabular}{|l|l|}
\hline \multicolumn{1}{|c|}{$\begin{array}{c}\text { Hallazgos } \\
\text { Visuales }\end{array}$} & \multicolumn{1}{c|}{ Etapa de la enfermedad } \\
\hline Agudeza Visual & $\begin{array}{l}\text { Etapa 1: Normal en muchos pacientes } \\
\text { Etapa 2 y 3: Alterada }\end{array}$ \\
\hline Visión del color & $\begin{array}{l}\text { Etapa 1, 2 y 3: 50\% de los pacientes } \\
\text { afectados en algunos estudios }\end{array}$ \\
\hline Campos Visuales & $\begin{array}{l}\text { Etapa 3: Pocos estudios. Campo } \\
\text { visual inferior afectado }\end{array}$ \\
\hline Estereopsis & $\begin{array}{l}\text { Etapa 3: Reducido en pacientes } \\
\text { visualmente sintomáticos }\end{array}$ \\
\hline
\end{tabular}

\begin{tabular}{|l|l|}
\hline Fijación & $\begin{array}{l}\text { Etapa 1, 2 y 3: Afectado en algunos } \\
\text { pacientes }\end{array}$ \\
\hline $\begin{array}{l}\text { Movimientos } \\
\text { oculomotores }\end{array}$ & $\begin{array}{l}\text { Etapa 2 y 3: Velocidad retrasada. 50\% } \\
\text { de los pacientes muestran anomalías } \\
\text { en la iniciación sacádica }\end{array}$ \\
\hline $\begin{array}{l}\text { Sensibilidad al } \\
\text { contraste }\end{array}$ & $\begin{array}{l}\text { Etapa 3: Puede ser un defecto que } \\
\text { afecta a todas las frecuencias }\end{array}$ \\
\hline $\begin{array}{l}\text { Percepción } \\
\text { Visoespacial }\end{array}$ & $\begin{array}{l}\text { Etapa 2 y 3: 40-50\% de los pacientes } \\
\text { muestran déficits }\end{array}$ \\
\hline Lectura & $\begin{array}{l}\text { Etapa 1, 2 y 3: Problemas para } \\
\text { entender las palabras escritas }\end{array}$ \\
\hline $\begin{array}{l}\text { Reconocimiento } \\
\text { de objetos }\end{array}$ & $\begin{array}{l}\text { Etapa 2 y 3: 50\% de los pacientes } \\
\text { revelan problemas con el } \\
\text { reconocimiento de objetos }\end{array}$ \\
\hline $\begin{array}{l}\text { Coordinación } \\
\text { ojo-cabeza }\end{array}$ & Alterada \\
\hline $\begin{array}{l}\text { Alucinaciones } \\
\text { Visuales }\end{array}$ & $\begin{array}{l}\text { Etapa 2 y 3: 20\% de los pacientes } \\
\text { experimentan alucinaciones visuales }\end{array}$ \\
\hline
\end{tabular}

Fuente. Elaboración autores, basado en Armstrong (57).

\section{Conclusiones}

- La AD genera alteraciones cognitivas como pérdida de la memoria, reducción del lenguaje, agnosia (dificultad para reconocer y asociar), apraxia (dificultad de ejecutar actos motores y voluntarios), además de alteraciones en las funciones visoespaciales.

- Las manifestaciones oculares inician con cambios estructurales en la retina al reducir el espesor de las células ganglionares de las capas de fibras nerviosas, en la microvasculatura retinal, la tortuosidad en arterias y venas frecuentemente asociadas con enfermedad vascular y envejecimiento, y al contener cuerpos de inclusión con proteína beta amiloide $A \beta$, donde se observan dursas y acumulos de placas seniles.

- Los principales hallazgos en nervio optico muestran neuropatía y esto se manifiesta como atrofia del disco óptico, aumento de la excavación patológica, adelgazamiento del borde neurorretiniano y de la capa de fibras nerviosas. 
- La agudeza visual y la sensibilidad al contraste se ven afectadas en estos pacientes, ya que los niveles de percepción de luminancia se reducen.

- Estos cambios visuales afectan la calidad de vida del paciente con $\mathrm{AD}$ pues presentan mayor riesgo de caídas y fracturas.

- Un estudio ocular profundo en $\mathrm{AD}$ es importante ya que la retina es la prolongación del cerebro, y a través de ésta se observan cambios asociados con la fisiopatología de la enfermedad sin intervenciones cerebrales invasivas.

\section{Referencias}

1. Patrick E, Rajagopal S, Wong H-KA, McCabe C, Xu J, Tang A, et al. Dissecting the role of non-coding RNAs in the accumulation of amyloid and tau neuropathologies in Alzheimer's disease. Mol Neurodegener [Internet]. 2017;12(1):51. Available from: http://molecularneurodegeneration.biomedcentral.com/articles/10.1186/s13024-017-0191-y

2. Naj AC, Jun G, Reitz C, Kunkle BW, Perry W, Park YS, et al. Effects of multiple genetic loci on age at onset in late-onset Alzheimer disease: a genome-wide association study. JAMA Neurol [Internet]. 2014;71(11):1394-404. Available from: http://www.ncbi.nlm.nih.gov/pubmed/25199842\%5Cnhttp://www.pubmedcentral.nih.gov/articlerender.fcgi?arti$\mathrm{d}=$ PMC4314944

3. Reitz C, Mayeux R. Alzheimer disease: Epidemiology, Diagnostic Criteria, Rist Factors and Biomarkers. Biochem Pharmacol. 2014;88(4):640-51.

4. Javaid FZ, Brenton J, Guo L, Cordeiro MF. Visual and ocular manifestations of Alzheimer's disease and their use as biomarkers for diagnosis and progression. Front Neurol. 2016;7(APR).

5. Hart NJ, Koronyo Y, Black KL, Koronyo-Hamaoui M. Ocular indicators of Alzheimer's: exploring disease in the retina. Acta Neuropathol [Internet]. 2016;132(6):767-87. Available from: https://doi.org/10.1007/s00401-016-1613-6

6. Lang A, Carass A, Al-Louzi O, Bhargava P, Solomon SD, Calabresi PA, et al. Combined registration and motion correction of longitudinal retinal OCT data. 2016;97840X. Available from: http://proceedings.spiedigitallibrary.org/ proceeding.aspx? $\mathrm{doi}=10.1117 / 12.2217157$

7. Haaksma ML, Vilela LR, Marengoni A, Caldero A, Leoutsakos JS, Rikkert MGMO. Comorbidity and progression of late onset Alzheimer's disease: A systematic review. 2017;(May):1-15.

8. Lopera F. La enfermedad de Alzheimer familiar. Desde la Bibl [Internet]. 2016;0(51):63-73. Available from: http://itmojs. itm.edu.co/index.php/desdelabiblioteca/article/view/915

9. Chao, MD, MSc*, J. Scott Roberts, PhD $\dagger$, Theresa M. Marteau, $\mathrm{PhD} \ddagger$, Rebecca Silliman, MD, $\mathrm{PhD}^{*}, \$, \mathrm{~L}$. Adrienne Cupples, PhD $\ddagger$, and Robert C. Green, MD M. Health Behavior Changes After Genetic Risk Assessment for Alzheimer Disease: The REVEAL Study. Alzheimer Dis Assoc Disord. 2008;100(2):130-4.

10. Vega GL, Weiner MF, Lipton AM, Von Bergmann K, Lutjohann D, Moore C, et al. Reduction in levels of 24S-hydroxycholesterol by statin treatment in patients with Alzheimer disease. Arch Neurol [Internet]. 2003;60(4):510-5. Available from: http://www.ncbi.nlm.nih.gov/pubmed/12707064

11. Leoni V. Oxysterols as markers of neurological disease - A review. Scand J Clin Lab Invest. 2009;69(1):22-5.

12. Pawlowski M, Meuth SG, Duning T. Cerebrospinal Fluid Biomarkers in Alzheimer's Disease-From Brain Starch to Bench and Bedside. Diagnostics [Internet]. 2017;7(3):42. Available from: http://www.mdpi.com/2075-4418/7/3/42

13. Thinakaran G, Koo EH. Amyloid precursor protein trafficking, processing, and function. J Biol Chem. 2008;283(44):29615-9.

14. Tabaton M, Piccini A. Role of water-soluble amyloid-beta in the pathogenesis of Alzheimer's disease. Int J Exp Pathol [Internet]. 2005;86(3):139-45. Available from: http://www. pubmedcentral.nih.gov/articlerender.fcgi? artid=2517412\&tool=pmcentrez\&rendertype $=$ abstract

15. Herrera-Rivero M, Hernández-Aguilar ME, Manzo J, Aranda-Abreu GE. Alzheimer â€TM s disease: Immunity and diagnosis Enfermedad de Alzheimer : inmunidad y diagnóstico. Rev Neurol. 2016;(November).

16. Chiasseu M, Alarcon-Martinez L, Belforte N, Quintero H, Dotigny F, Destroismaisons L, et al. Tau accumulation in the retina promotes early neuronal dysfunction and precedes brain pathology in a mouse model of Alzheimer's disease. Mol Neurodegener [Internet]. 2017;12(1):58. Available from: http://molecularneurodegeneration.biomedcentral. com/articles/10.1186/s13024-017-0199-3

17. Custodio N, Montesinos R, Alva C, Mejía K, Becerra Y, Lira D. Nuevos términos clínicos, prevención y tratamiento del trastorno cognitivo vascular: revisión de literatura basada en la evidencia. Rev Neuropsiquiatr [Internet]. 2016;79(3):152-65. Available from: http://www.scielo.org. $\mathrm{pe} / \mathrm{pdf} / \mathrm{rnp} / \mathrm{v} 79 \mathrm{n} 3 / \mathrm{a} 04 \mathrm{v} 79 \mathrm{n} 3 . \mathrm{pdf}$

18. Minsalud. Informe al Congreso de la República 2014-2015. Minist Salud y Protección Soc [Internet]. 2015;194. Available from: https://www.minsalud.gov.co/sites/rid/Lists/BibliotecaDigital/RIDE/DE/PES/informe-congreso-2014-2015.pdf

19. Armstrong $\mathrm{R}$ a. The molecular biology of senile plaques and neurofibrillary tangles in Alzheimer's disease. Folia Neuropathol. 2009;47(4):289-99.

20. Sánchez CR De, Nariño D, Fernando J, Cerón M. Epidemiología y carga de la Enfermedad de Alzheimer. Acta Neurológica Colomb. 2010;26(3):87-94. 
21. Hurtado AMA, Quintana ASSR. Relación entre la calidad de vida en salud y la carga física en cuidadores de personas con enfermedad de Alzheimer. Rev Colomb Salud Ocup. 2016;6(1):18-23.

22. Carvajal-castrillón J, Marina L, Toro G, Estrada DO, Ramírez AA, Peláez AR, et al. Prevalencia de síndromes neuropsicológicos del adulto en una unidad de neuropsicología en Medellín, Colombia Prevalence of adult neuropsychological syndromes in a neuropsychology unit. Acta Neurológica Colomb. 2015;31(1):20-6.

23. Batsch NL, Mittelman MS. Overcoming the stigma of dementia, World Alzheimer Report 2012 [Internet]. Www.Alz. Co.Uk. 2012. 1-80 p. Available from: papers2://publication/ uuid/889ADA69-3B6E-4DCC-BF40-936650E90072

24. Bateman RJ, Xiong C, Benzinger TLS, Fagan AM, Goate A, Fox NC, et al. Clinical and Biomarker Changes in Dominantly Inherited Alzheimer's Disease. N Engl J Med [Internet]. 2012;367(9):795-804. Available from: http://www. nejm.org/doi/abs/10.1056/NEJMoa1202753

25. Crous-Bou M, Minguillón C, Gramunt N, Molinuevo JL. Alzheimer's disease prevention: from risk factors to early intervention. Alzheimers Res Ther [Internet]. 2017;9(1):71. Available from: http://alzres.biomedcentral.com/articles/10.1186/s13195-017-0297-z

26. Ji F, Pasternak O, Liu S, Loke YM, Choo BL, Hilal S, et al. Distinct white matter microstructural abnormalities and extracellular water increases relate to cognitive impairment in Alzheimer's disease with and without cerebrovascular disease. Alzheimers Res Ther [Internet]. 2017;9(1):63. Available from: http://alzres.biomedcentral.com/articles/10.1186/ s13195-017-0292-4

27. Croteau E, Castellano CA, Fortier M, Bocti C, Fulop T, Paquet $\mathrm{N}$, et al. A cross-sectional comparison of brain glucose and ketone metabolism in cognitively healthy older adults, mild cognitive impairment and early Alzheimer's disease. Exp Gerontol [Internet]. 2017; Available from: http://linkinghub.elsevier.com/retrieve/pii/S0531556517302280

28. Epperly T, Dunay MA, Boice JL. Alzheimer Disease: Pharmacologic and Nonpharmacologic Therapies for Cognitive and Functional Symptoms. Am Fam Physician. 2017;95(12):771-8.

29. Tam WK, Chan H, Brown B, Leung KW, Woo V, Yap M. Aging and mfERG topography. Eye. 2006;20(1):18-24.

30. Hollands C, Tobin MK, Hsu M, Musaraca K, Yu T-S, Mishra R, et al. Depletion of adult neurogenesis exacerbates cognitive deficits in Alzheimer's disease by compromising hippocampal inhibition. Mol Neurodegener [Internet]. 2017;12(1):64. Available from: http://molecularneurodegeneration.biomedcentral.com/articles/10.1186/s13024-0170207-7

31. Chan D, Gallaher LM, Moodley K, Minati L, Burgess N, Hartley T. The 4 Mountains Test: A Short Test of Spatial Memory with High Sensitivity for the Diagnosis of Pre-dementia Alzheimer's Disease. J Vis Exp [Internet]. 2016;(116). Available from: http://www.jove.com/video/54454/the-4- mountains-test-short-test-spatial-memory-with-high-sensitivity

32. Bruni BM, Granado FB, Prado RA. Avaliação do equilíbrio postural em idosos praticantes de hidroterapia em grupo. $\mathrm{O}$ Mundo da Saúde. 2008;32(1):56-63.

33. Mar J, Soto-Gordoa M, Arrospide A, Moreno-Izco F, Martínez-Lage P. Fitting the epidemiology and neuropathology of the early stages of Alzheimer's disease to prevent dementia. Alzheimers Res Ther [Internet]. 2015;7(1):2. Available from: http://alzres.com/content/7/1/2

34. EC M, KV P. Cognitive decline in preclinical stage 2 alzheimer disease and implications for prevention trials. JAMA Neurol [Internet]. 2016 Jun 1;73(6):640-2. Available from: http://dx.doi.org/10.1001/jamaneurol.2016.0281

35. TRABAJO FIN DE GRADO TÍTULO : " BIOMARCADORES EN LA ENFERMEDAD DE ALZHEIMER .2016:1-22.

36. Ma D, Alonso E, Ana D, Sosa L, Sotelo J, Ausman J, et al. Visión actual de las demencias. Arch Neurociencias. 2016;21 (Suplemento Especial):1-8.

37. Baker JE, Lim YY, Pietrzak RH, Hassenstab J, Snyder PJ, Masters CL, et al. Cognitive impairment and decline in cognitively normal older adults with high amyloid- $\beta$ : A meta-analysis. Alzheimer's Dement Diagnosis, Assess Dis Monit [Internet]. 2017;6:108-21. Available from: http://dx.doi. org/10.1016/j.dadm.2016.09.002

38. TL K, Goldacre R, MJ G. Associations between age-related macular degeneration, alzheimer disease, and dementia: Record linkage study of hospital admissions. JAMA Ophthalmol [Internet]. 2014 Jan 1;132(1):63-8. Available from: http://dx.doi.org/10.1001/jamaophthalmol.2013.5696

39. Williams MA, McGowan AJ, Cardwell CR, Cheung CY, Craig D, Passmore P, et al. Retinal microvascular network attenuation in Alzheimer's disease. Alzheimer's Dement Diagnosis, Assess Dis Monit [Internet]. 2015;1(2):229-35. Available from: http://dx.doi.org/10.1016/j.dadm.2015.04.001

40. Schwitzer T, Schwan R, Bubl E, Lalanne L, Angioi-Duprez $\mathrm{K}$, Laprevote $\mathrm{V}$. Looking into the brain through the retinal ganglion cells in psychiatric disorders: A review of evidences. Prog Neuro-Psychopharmacology Biol Psychiatry [Internet]. 2017;76(January):155-62. Available from: http://dx.doi.org/10.1016/j.pnpbp.2017.03.008

41. den Haan J, Verbraak FD, Visser PJ, Bouwman FH. Retinal thickness in Alzheimer's disease: A systematic review and meta-analysis. Alzheimer's Dement Diagnosis, Assess Dis Monit [Internet]. 2017;6:162-70. Available from: http://linkinghub.elsevier.com/retrieve/pii/S2352872916300793

42. Pillai JA, Bermel R, Bonner-Jackson A, Rae-Grant A, Fernandez $\mathrm{H}$, Bena J, et al. Retinal Nerve Fiber Layer Thinning in Alzheimer's Disease. Am J Alzheimer's Dis Other Dementiasr [Internet]. 2016;31(5):430-6. Available from: http:// journals.sagepub.com/doi/10.1177/1533317515628053

43. Coppola G, Di Renzo A, Ziccardi L, Martelli F, Fadda A, Manni G, et al. Optical coherence tomography in Alzhei- 
mer's disease: A meta-analysis. PLoS One. 2015;10(8):1-14.

44. Ong YT, Hilal S, Cheung CY, Venketasubramanian N, Niessen WJ, Vrooman H, et al. Retinal neurodegeneration on optical coherence tomography and cerebral atrophy. Neurosci Lett. 2015;584:12-6.

45. Khawaja AP, Chan MPY, Yip JLY, Broadway DC, Garway-Heath DF, Luben R, et al. Retinal Nerve Fiber Layer Measures and Cognitive Function in the EPIC-Norfolk Cohort Study. Investig Opthalmology Vis Sci [Internet]. 2016;57(4):1921. Available from: http://iovs.arvojournals.org/article.aspx?doi $=10.1167 /$ iovs. $16-19067$

46. La Morgia C, Ross-Cisneros FN, Koronyo Y, Hannibal J, Gallassi R, Cantalupo G, et al. Melanopsin retinal ganglion cell loss in Alzheimer disease. Ann Neurol. 2016;79(1):90-109.

47. Pavisic IM, Firth NC, Parsons S, Rego DM, Shakespeare TJ, Yong KXX, et al. Eyetracking metrics in young onset alzheimer's disease: A Window into cognitive visual functions. Front Neurol. 2017;8(AUG):1-16.

48. Kirby E, Bandelow S, Hogervorst E. Visual Impairment in Alzheimer's Disease: A Critical Review. Vol. 21, Journal of Alzheimer's disease : JAD. 2010. 15-34 p.

49. Pal S, Sanyal D, Biswas A, Paul N, Das SK. Visual Manifestations in Alzheimer's disease: A Clinic-Based Study From India. Am J Alzheimer's Dis Other Dementiasr [Internet]. 2013 Jul 2;28(6):575-82. Available from: https://doi. org/10.1177/1533317513494448

50. García EP, Echevarría IOH, Rodríguez IY. Obtención , interpretación y usos del electrorretinograma multifocal Obtaining , interpreting and using the multifocal electroretinogram. 2016;29(1):105-23.

51. Bernal Luz Mery, López Greizy. Diagnóstico prénatal: retrospectiva. Nova. 2014; 12( 21 ): 23-36.

52. Márquez Gómez Marco Antonio, Gómez Díaz Graciela María. Accidente ofídico en el departamento de Sucre, Colombia. Nova. 2015; $13(24)$ : 39-46.

53. González Yuri Lilian. Evaluación de la percepción del riesgo ocupacional en trabajadores de una empresa del sector de la construcción en Bogotá D.C. Nova. 2015; 13 ( 23 ): 93-107.

54. Naranjo Flórez Ricardo Andrés. Avances y perspectivas en Síndrome de Asperger. Nova. 2014; 12( 21 ): 81-101.

55. Almonacid Urrego Carmen Cecilia, Camarillo Romero María del Socorro, Gil Murcia Zulay, Medina Medina Claudia Yasmin, Rebellón Marulanda Jennifer Viviana, Mendieta Zerón Hugo. Evaluación de factores de riesgo asociados a enfermedad cardiovascular en jóvenes universitarios de la Localidad Santafé en Bogotá, Colombia. Nova. 2016; 14(25): 9-17.

56. González Devia Johanna L., Monroy Romero Paola A., Almonacid Urrego Carmen C.. Homocisteína y otros factores de riesgo cardiovascular en niños de educación básica primaria del Colegio Distrital Manuel Elkin Patarroyo, Bogotá, D.C. Colombia. Estudio piloto. Nova. 2017 ; 15( 27 ): 103-117.

57. Armstrong RA, Syed AB. Alzheimer's disease and the eye. Ophthalmic Physiol Opt [Internet]. 2009;16(SUPPL.
1):103-11. Available from: http://dx.doi.org/10.3921/joptom. 2009.103

58. Sivak, J. The Aging Eye: Common Degenerative Mechanisms Between the Alzheimer's Brain and Retinal Disease. Investigative Ophthalmology \& Visual Science. [Internet] 2013, Vol. 54, No. 1. Available from: doi:10.1167/iovs.1210827 